\title{
Candida infections: Novel virulence factors and mechanisms of azole resistance
}

\author{
Krishnaswamy Nalina \\ Department of Medical Microbiology, Universiti of Kuala Lumpur Royal College of Medicine, No. 3, Jalan Green Town, \\ Perak Darul Ridzuan, Ipoh 30450, Malaysia. \\ Email:knalina@rcmp.unikl.edu.my
}

Received 21 October 2013; Received in revised form 24 March 2014; Accepted 5 April 2014

\begin{abstract}
Candida represents the most common cause of fungal infections in the immunocompromised host. The incidence of lifethreatening fungal infections due to Candida spp. has markedly increased over the past two decades. Antifungal treatment of candida infections is hampered by several factors, including limited number of active agents, emergence of refractory fungal species, and development of antifungal drug resistance. Here we reviewed the infections caused by Candida spp. and virulence factors that facilitate disease pathogenesis in the immunocompromised host. Aside from this, a detailed discussion on the different mechanisms of azole resistance has also been presented, to better understand and design better strategies against drug-resistant candida to improve the quality of life of individuals.
\end{abstract}

Keywords: Azole; Candida tropicalis; Genotypes; Resistance.

\section{INTRODUCTION}

The research on the biology of medically important fungi has increased dramatically, and more recently has entered into an era of genomics. Numerous factors contribute to increased incidence of fungal infections, especially defective CD4+ T cells functions, metabolic dysfunction and chronic debilitation, increased use of broad-spectrum antibiotics, cytotoxic chemotherapeutics, and solid organ/bone marrow transplantations (Groll et al., 2001; Sanglard and Bille, 2002). In addition, environmental exposures to a variety of endemic fungal pathogens are common, which increases the risk of acquiring fungal infections in elderly individuals (Pfaller et al., 2007). Fungal infections may be divided into two broad categories, viz., nosocomial and community-acquired, the former commonly being opportunistic mycoses, which at times cause life-threatening infections in immunocompromised individuals (Shankar et al., 2006; Shankar et al., 2007). While community-acquired infections encompasses endemic agents, such as Blastomyces dermatidis, Coccidioides immitis, Histoplasma capsulatum, Paracoccidioides brasiliensis, and Penicillium marneffei (Wenzel et al., 2003), opportunistic pathogens include Candida spp, Aspergillus spp, Cryptococcus neoformans, and Pseudallescheria boydii. One report suggests the role of certain uncommon species such as Mucor spp., Rhizopus spp., Fusarium spp. and Acremonium spp. in immunocompromised subjects (Groll et al., 2001). Candida consists of nearly 200 species of which only few are associated with human infections.
Initially, although it was believed that $C$. albicans was the only species of medical importance, there was a shift during the 1980's, in the incidence of other species viz. C. tropicalis, C. guilliermondii, C. glabrata, C. krusei and C. lusitaniae. C. tropicalis causing severe invasive disease associated with a high mortality rate (Gary et al., 2002). Antifungal treatment of candida infections is hampered by several factors, including limited number of active agents, emergence of refractory fungal species, and development of antifungal drug resistance. Here we reviewed the infections caused by candida and virulence factors that facilitate disease pathogenesis in the immunocompromised host. Aside from this, a detailed discussion on the different mechanisms of azole resistance has been discussed to better understand and design effective strategies against drug-resistant candida to improve the quality of life of infected individuals.

\section{I) Candida infections}

Candida spp. represents the most frequent cause of fungal infections in the immunocompromised host although considered part of the normal flora in a healthy host. Recovery of an organism from a normally sterile site or from several different body sites is an indicator of disseminated infection with probable fungaemia. Candida spp cause a wide spectrum of disease ranging from thrush to invasive diseases (Groll et al., 2001). Infections of the skin and mucosa due to Candida spp. may occur in both immunocompromised and immunocompetent individuals. Impaired host immunity is one of the critical factors that transform candida from normal flora to a 
pathogen. The cutaneous infection may range from oral colonization to chronic candidiasis. The reduced CD4+ T cell counts in HIV infection, radioactive therapy for head and neck cancer, reduced saliva, prolonged antibiotic therapy, diabetes and gastrointestinal infections are other predisposing factors. Clinical presentations range from erythematous, hyperplastic, pseudomembraneous candidiasis to angular chelitis (Runkhe, 2002). Esophageal candidiasis presents with dysphagia or odynophagia leading to ulceration or erosion of the esophagus. Vulvovaginal candidiasis is a mucosal infection caused commonly by $C$. albicans especially in the female genital mucosa (Runkhe, 2002). Other manifestation includes skin rashes and paronychia. Onychomycosis is uncommon unless it occurs in patients with chronic mucocutaneous candidiasis, and represents the initial manifestation in patients with HIV infection (Groll et al., 2001).

Candidemia refers to recovery of candida from at least one blood culture specimen. The infections are invasive, may involve one or multiple organs or sometimes hematogenously disseminated (Michael and Edmond, 1999). Majority of disseminated infections originates from the gastrointestinal tract, especially after gastrointestinal surgery and intra-abdominal sepsis. Patients with leukemia or lymphoma receiving chemotherapy may experience colonization and eventual systemic invasion (Bernad and Naglik, 2002). Disseminated candidiasis represents one of the major causes of morbidity and mortality among neutropenic patients. $C$. albicans is the most frequent cause of candidemia in patients with solid tumors. However, there is an increase in the prevalence of NAC species of Candida, especially C. tropicalis predominantly among patients with hematologic malignancies (Sanglard and Bille, 2002). The combination of prolonged immunosuppressive therapy renders solidorgan transplant recipients at high risk for developing disseminated candidiasis. Prolonged immunosuppression enhances the susceptibility to colonization of mucosal surfaces with Candida spp., which leads to invasion of blood stream. The incidence of candidemia is higher in liver transplant patients than renal transplants. A substantial percentage of candida infections in transplant recipients may originate from the donor organ. $C$. albicans and $C$. tropicalis accounts for most candidal infections in the organ transplant patient (Carlos and Paya, 1993). Patients with HIV infection who developed disseminated candidiasis have additional risk factors such as indwelling central catheters, parenteral nutrition, use of broad spectrum antibiotics or neutropenia or cytotoxic therapy, burns and low birth weight (Walsh, 1995). Both burn patients and neonates have neutrophil dysfunction, which increases susceptibility to disseminated candidiasis. Although $C$. albicans remains the most important cause of disseminated candidiasis, the incidence of $C$. tropicalis, $C$. glabrata, and $C$. krusei, has been steadily increasing over the years. C. albicans accounts for $50-55 \%$ blood-stream isolates; C. tropicalis and C. glabrata for $10-20 \%$ and C. krusei for $2-4 \%$ of the isolates (Kullberg and Filler, 2002). Non-albicans candida
Non-albicans candida (NAC) is an emerging cause of nosocomial systemic fungal infections. The most common NACs are C. tropicalis, C. glabrata, C. krusei and C. parapsilosis, which as a group represent about one-half of all NAC species isolated from blood. Fungaemia with or without complications due to NAC represents a special concern owing to the virulence and pathogenicity of some species, particularly in immunocompromised hosts, and the emergence of antifungal drug resistance. The proportion NAC species is increasing over the last two decades. In 1990, NAC represented $10-40 \%$ of all cases of candidemia; although in 1998, there was a steady rise $(35-65 \%)$. The most common NACs are C. parapsilosis (20-40\%); C. tropicalis (10-30\%); C. krusei (10-35\%); and C. glabrata (5-40\%). However, there are differences in both overall and attributable mortality among species: The lowest mortality is associated with $C$. parapsilosis, the highest with $C$. tropicalis and $C$. glabrata. Mortality in NAC species appears to be highest in ICU and surgical patients and lowest in cancer patients, children and HIV positive patients. C. tropicalis may be present in the oral cavity of asymptomatic carriers, and could be associated with fungemia, post surgical infections, osteomyelitis, and endocarditis. Endogenous infections due to $C$. tropicalis are most common (Kullberg and Filler, 2002).

C. tropicalis is pathogenic yeast, present in the oral cavity of asymptomatic carriers. It has been associated with various clinical conditions such as fungemia, postsurgical infections, osteomyelitis, endocarditis, meningitis and neonatal infections (Wingard, 1995). C. tropicalis is reportedly the most frequently encountered NAC causing systemic disease in oncology units. It is an important cause of disseminated disease in patients with underlying malignancies and allogeneic bone marrow transplantation (BMT). C. tropicalis fungemia is also common in leukemia (Kontoyiannis et al., 2001). C. tropicalis arthritis has been reported in patients with lymphoblastic leukemia (Sim et al., 2005). Eventhough $C$. albicans is the most commonly recovered species from the oral cavity of HIV patients, $C$. tropicalis is frequently recovered in advanced AIDS who are under prolonged azole treatment (Michael, 2003). In one study in India, $C$. tropicalis was the predominant isolate in blood-stream infections (Mary et al., 2001).

C. tropicalis has also been reported to cause osteomyelitis in BMT cases (Christelle and Bradley, 1994). A case of $C$. tropicalis in sternal wound infection has also been observed in one study (Bradly and Richard, 1991). C. tropicalis has been considered to be more virulent in patients with disrupted mucosal integrity (Peter et al., 2006). Candida meningitis is rare, but has been increasingly recognized in recent years. It can occur spontaneously as a complication of disseminated candidiasis or as a complication of surgery. Most cases of meningitis are complications of disseminated candidiasis. C. tropicalis is the second most prevalent species other than C. albicans in meningitis (Nguyen et al., 1995). Candidal colonization of the respiratory tract is common in patients receiving mechanical ventilation. $C$. tropicalis was isolated from $13.1 \%$ of cases with underlying respiratory 
ailments and on mechanical ventilation (Azoulay et al., 2006). C. tropicalis also occurs in neonatal intensive care units (NICUs), gynecological and ophthalmic conditions, and infective endocarditis (Montgomerie, 1993; Gerritsen et al., 1998; Dorko et al., 2000; Roilides et al., 2003).

\section{II) Virulence factors and pathogenesis}

Candida reportedly produces a plethora of virulence factors in the host to facilitate invasive disease in the host. Synthesis of hydrolase is known to play a central role in candida pathogenesis. The production of hydrolytic enzymes may account for a number of pathogenic functions in addition to the simple role of digesting molecules for nutrient acquisition. Candida spp. produce hydrolases with broad substrate specificities viz., aspartic proteinases, phospholipases and lipases (Bernad and Naglik, 2002).

\section{Secreted Aspartyl Proteinases (SAP)}

Extracellular proteinase activity of candida is due to a single class of proteinases, such as secreted aspartic proteinases. Ten SAP genes that exhibit broad substrate specificity encode the proteinase family, which degrade many human proteins such as albumin, haemoglobin, keratin, collagen, mucin, lactoferrin, lactoperoxidase and secretory IgA. Many of the pathogenic species possess $S A P$ genes, including $C$. tropicalis, $C$. dubliniensis and $C$. parapsilosis, and produce active extracellular proteinases in vitro. The proteinase activity differs with $\mathrm{pH}$. Different $S A P$ genes appear to be essential for mucosal (SAP1 $S A P 3)$ and systemic (SAP4 - SAP6) infections, which are involved in adherence, tissue damage and evasion of host immune responses (Cassone et al., 1999). Sap 1-3 (yeast-associated) has optimum activity at lower $\mathrm{pH}$ values; similarly sap 4-6 (hyphal-associated) has optimum activity at higher $\mathrm{pH}$ values. Sap 2 has been shown to be stable and active at neutral $\mathrm{pH}$. Less pathogenic or nonpathogenic Candida spp. do not produce significant levels of proteinase, even though they possess aspartyl proteinase genes. Numerous studies have proven the role of Sap as a potential virulence factor. The proteolytic candida adheres more readily to buccal epithelial and endothelial cells than less proteolytic strains. Proteinase producing candida are relatively more invasive (Bernardis et al., 1996).

\section{Phospholipases (PL)}

Phospholipids and proteins represent the major chemical constituents of the host cell envelope. Phospholipases designated as PLA, PLA2, PLB, PLC, and PLD, are heterogenous enzymes, which hydrolyze ester linkages present in phospholipids. Invasion of host cell by microbes entails penetration that causes damage to the outer cell envelope, and is mediated by either physical or enzymatic means or a combination of both. Phospholipases and proteinases are involved in the membrane disruption processes, which occur during host cell invasion (Price et al., 1982; Ghannoum, 2000;). It was initially believed that only C. albicans and not other species, produce extracellular phospholipase. In contrast, others recently showed that NACs namely C. glabrata (41\%), $C$. parapsilosis $(50 \%)$, C. tropicalis $(70 \%)$, C. lusitaniae $(80 \%)$ and C. krusei $(100 \%)$ produce detectable phospholipase, and the role of candidal phospholipases in virulence has shown a clear correlation with pathogenicity in mice.

\section{Esterases}

Microbial lipases such as esterase serve as a potential virulence factor in severe infections. During infection, secreted lipases might support fungal growth and colonization through cleavage of sebum-derived triacylglycerols (Stehr et al., 2003). Tween acts as a substrate, which could be used to ascertain the patterns of lipolytic activity of clinically significant candida isolates. The presence of a halo around the site of inoculation indicates a positive test for esterase activity (Rudek, 1978; Slifkin, 2000).

\section{Biofilms and drug resistance}

Biofilms are universal, complex, interdependent communities of surface-associated microorganisms enclosed in an exopolysaccharide matrix occurring on any surface. In medical devices, they grow as structured biofilms, and are developed from a single species or a community derived from multiple species. Ecologic advantages of forming a biofilm include protection from adverse environments, nutrient availability, metabolic cooperation and acquision of new genetic traits. Biofilms are notoriously difficult to eliminate and are a source of many recalcitrant infections. A variety of microbial infections are caused by biofilms ranging from urinary tract and catheter-borne infections, otitis media in children and dental plaques, to life-threatening endocarditis. Immunocompromised patients are more susceptible than immunocompetent (Ramage et al., 2001; Ramage et al., 2002; Jabra et al., 2004).

In oral or pharyngeal infections, yeast cells must initially adhere to oral epithelial cells or dentures or coaggregate with other oral flora. The detailed structure of a mature $C$. albicans biofilm consists of a dense network of yeasts, hyphae and matrix. However, it is not observed when the organism is grown in liquid culture or on agar. Interestingly, bacteria are often found in association with candida in biofilms in vivo, indicating extensive interspecies interactions (Jyotsna et al., 2001). Biofilm producing systemic isolates are more common among NACs $(93.1 \%)$ in comparison to C. albicans $(42.9 \%)$. Of the NACs, C. tropicalis showed the highest relative intensity $(54.17 \%)$ of biofilm production (Girish Kumar and Thangam, 2006). The consequences of biofilm growth with profound clinical implications are enhanced resistance to antimicrobial agents and protection from 
host defenses. Hence, biofilm-associated infections are frequently refractory to conventional therapy.

C. albicans and other closely related Candida spp. are now recognized as major agents of nosocomial infection worldwide, especially, nosocomial pneumonia and urinary tract infections. Almost invariably, an implanted device such as an intravascular or urinary catheter or endotracheal tube is associated with these infections and a biofilm can be detected on the surface of the device. Other implants such as prosthetic heart valves, cardiac pacemakers and joint replacements (hip, knee etc) are also susceptible to infection that usually occurs at the time of surgery. At times, infusion fluids, or catheter hubs are contaminated with skin microflora or iatrogenically. The most significant feature of microbial biofilms is resistance to myriad antimicrobial agents first demonstrated by Hawser in 1995. Antifungal agents such as amphotericin $\mathrm{B}$, fluconazole, flucytosine, itraconazole and ketoconazole appear to show less activity against $C$. albicans biofilms (Hawser and Douglas, 1995). The potential mechanisms of resistance include: a) restricted penetration across biofilm matrix, b) phenotypic changes resulting from decreased growth rate or nutrient limitation, c) surfaceinduced expression of resistance genes, and d) 'persister' cells (Julia, 2002).

\section{III) Antifungal agents and resistance}

Antifungal treatments against Candida infections are hampered by several factors, including the limited number of active agents, emergence of refractory fungal species, and development of resistance, which has triggered pursuit for newer agents with novel modes of action. Antifungal agents have targeted different cellular processes involved in fungal biosynthesis (Sanglard and Bille, 2002). In general, antifungal agents interact with ergosterol, the major component found on fungal cell membrane that contributes to a variety of cellular functions. It is important for membrane fluidity and integrity for the function of numerous membrane-bound enzymes, including chitin synthetase implicated with proper cell growth and division (White et al., 1998). A list of common antifungal agents is shown in Table 1.

\section{Polyenes}

Polyenes are a natural broad-spectrum antifungal compound discovered in the early 1950's. Important polyenes are amphotericin B (AMB), nystatin and natamycin. AMB is produced by Streptomyces nodosus, and is amphipathic having both hydrophobic and hydrophilic sites. AMB binds with ergosterol in the cell membrane forming a channel through which cellular components leak, leading to increased membrane permeability, destruction and cell death (Nafsika et al., 1996; White, 1998;). High concentrations of AMB can damage erythrocytes and other mammalian cells and cause osmotic leakage of hemoglobin and intracellular ions. Systemic and renal manifestations are often encountered with $A M B$. To alleviate the side-effects, $A M B$ is often incorporated into lipid vehicles (Abelect), in liposomes (Ambisome) and in colloidal suspensions (Amphotec) (Sanglard and Bille, 2002).

\section{Mechanism of AMB resistance}

Any alteration in the sterol content of fungal cell that influences the availability of ergosterol could result in AMB resistance (Loeffler and Stevens, 2003). The quantitative mechanisms include decrease of ergosterol due to lack of synthesis, and replacement with episterol, fecosterol or other sterols (Cheng et al., 2006). The qualitative mechanism includes reorientation or membrane masking, which could result in less-favored binding with AMB. The other factor related to AMB resistance is the growth phase of the fungal cell (Seneviratne et al., 2008). During exponential growth, breakdown and resynthesis of cell wall occurs at a high rate, providing enhanced access of AMB to the cell membrane. At stationary growth phase, breakdown and synthesis of the cell wall occurs at a much lower rate, leading to relative resistance to AMB (Cannon et al., 2009). Previous exposure to azoles leading to depletion of ergosterol is also responsible for AMB resistance (Cannon et al., 2009). Molecular mechanism of resistance involves two genes, ERG2 and ERG3. Several enzymes take part in the chain of reactions starting from squalene and ending up with ergosterol synthesis (Arikan and Rex, 2005). In this pathway, mutation in two genes encoding two enzymes has been found to be associated with $\mathrm{AMB}$ resistance. $\mathrm{C}-8$ sterol isomerase catalyses the production of episterol from fecosterol regulated by the $E R G 2$ gene. C-5 sterol desaturase on the other hand, is one of the enzymes that convert episterol, encoded by the ERG3 gene to ergosterol (Munn et al., 1999). Clinical isolates of $\mathrm{AMB}$ resistant Candida spp. with reduced ergosterol and defective ERG2/ERG3 have been reported (Arikan and Rex, 2005).

\section{Cyclic lipopeptides}

Echinocandins are fatty acid derivatives of cyclic hexapeptides (Eschenauer et al., 2007). Caspofungin is the first commercialized water-soluble echinocandin derivative available only in parenteral form (Hashimoto, 2009). It exert antifungal activity via non-competitive inhibition of 1,3 beta-D-glucan, an essential cell wall homopolysaccharide present in several pathogenic fungi (Hashimoto, 2009). Glucan synthase is a heteromeric enzyme complex composed of two subunits (Martín et al., 2003), the large integral membrane protein referred to as FKS1 or FKS2 (encoded by FKS1 and FKS2 genes which are highly homologous) and the small subunit more loosely associated with the membrane named RHO1 (Garcia-Effron et al., 2009). Mutations in FKS1 and FKS2 genes results in caspofungin resistance (Arikan and Rex, 2005). 


\section{Azoles}

The azole derivatives discovered in the late 1960's are totally synthetic and are the most rapidly expanding antifungal compounds classified on the basis of presence of nitrogens in the five-membered azole ring (Nafsika et al., 1996). They are classified as imidazoles (ketoconazole, miconazole, clotrimazole) or triazoles (itraconazole, fluconazole and voriconazole). Imidazoles are limited to the treatment of superficial mycoses but triazoles in the treatment of both superficial and systemic fungal infections. The azoles target lanosterol demethylase, a cytochrome $\mathrm{P}_{450}$ enzyme containing a heme moiety in its active site in the ergosterol pathway. There are two nitrogen molecules present in the azoles. One nitrogen molecule binds to the iron atom of the heme preventing the demethylation of lanosterol. The other nitrogen interacts directly with the apoprotein of lanosterol demethylase. The position of this second nitrogen molecule in relation to the apoprotein may determine the specificity of different azole drugs for the enzyme. At high concentrations, the azoles may also interact directly with lipids in the membranes. The resulting ergosterol depletion and accumulation of lanosterol and other 14methylated sterols interfere with the 'bulk' functions of ergosterol as a membrane component; they disrupt the plasma membrane, making it more vulnerable to further damage, and alter the activity of membrane-bound enzymes, such as those associated with nutrient transport and chitin synthesis (White et al., 1998).

Azole antifungal agents are generally free of serious toxicity; however, rare cases of fatal hepatotoxicity have been reported. Because of their ability to inhibit the cytochrome $\mathrm{P}_{450}$ dependent enzymes involved in the biosynthesis of steroid hormones in mammalians cells, azole antifungal agents may produce endocrine-related side-effects, such as depletion of testosterone and glucocorticoids, resulting in gynecomastia and adrenal insufficiency, respectively (Nafsika et al., 1996). In order to increase the efficacy of azoles, several new derivatives eg. voriconazole, posaconazole and ravuconazole, are currently being developed and studied.

\section{Mechanisms of azole resistance}

Reduction in cellular drug import or reduced intracellular azole accumulation

Intracellular accumulation of azoles can be reduced by the lack of drug penetration because of low ergosterol levels or possibly decreased ratio between phosphatidyl-choline and phosphatidyl-ethanolamine in the plasma membrane, which may change membrane barrier functions. Drug import may also be affected by the sterol composition of the cell membrane. Several studies have demonstrated that when the ergosterol component is eliminated or reduced in favour of other sterol components, there are concomitant permeability changes in the cell membrane and a lack of fluidity, which could lower the capacity of azole drugs to enter the cell. Molecular analysis of the azole resistant strains has revealed the involvement of efflux plumps necessary to decrease accumulation of azoles (Figure 1) (Lupetti et al., 2002).

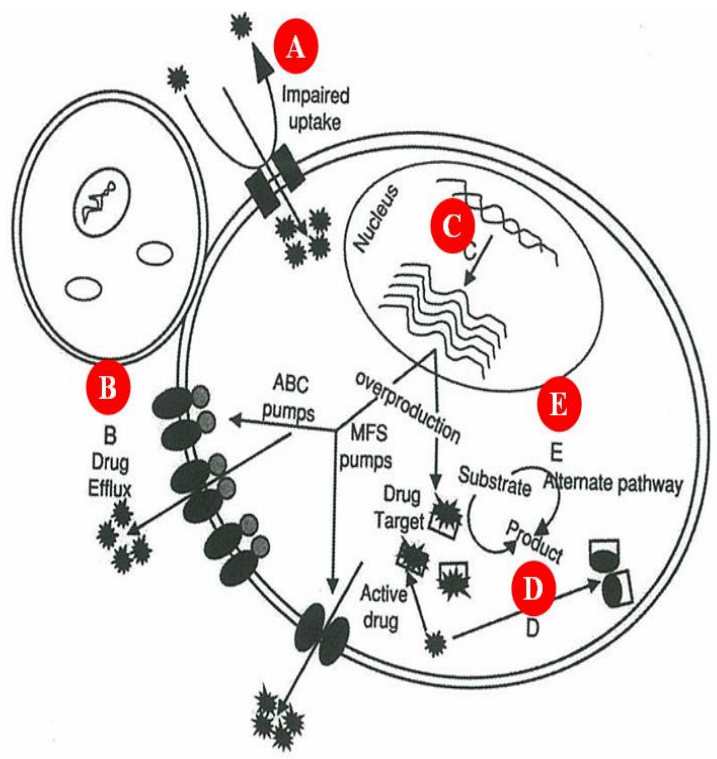

Figure 1: Mechanisms of azole resistance in Candida spp.: A, Reduced drug uptake (import) due to compositional changes in the cell wall or plasma membrane, B, Rapid efflux of drugs mediated by the ABC (ATP binding cassette) or MFS (Major facilitator superfamily) transporters; C, Overexpression of the genes encoding drug target ERG11, CDR1 and MDR1; D, Mutation in drug target; E, Activation of alternate ergosterol pathways.

\section{Alterations in ergosterol biosynthetic pathway}

Modification of the target enzyme and or other enzymes in the biochemical pathway is another common mechanism of azole resistance (Table 2). Azole drugs target the ergosterol pathway. The predominant azole target enzyme is lanosterol demethylase encoded by ERG 11 (previously referred as ERG16/CYP51A1). Genetic alterations in ERG11, including point mutations, overexpression, and gene amplification leading to gene expression, gene conversion and mitotic recombination have been observed in C. albicans and it is related to azole resistance. In addition to alterations in the lanosterol demethylase, common mechanism of azole resistance is an alteration in other enzymes in the same biosynthetic pathway. There are many numbers of genes encoding various enzymes in the ergosterol pathway namely ERG1 to ERG7, ERG11, ERG25, ERG26 and ERG27. Alterations in ERG3 and $E R G 11$ reportedly lead to azole resistance. ERG3 defects prevent the production of the diol that would cause growth arrest (White, 1998).

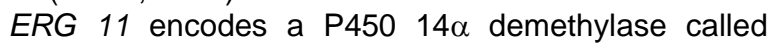
Erg11p, and point mutations in ERG11 leads to azole resistance. A point mutation leading to replacement of 
Table 1: Common antifungal agents and mode of action.

\begin{tabular}{|c|c|c|}
\hline Antifungal agents & Mode of action & Mechanism of resistance \\
\hline $\begin{array}{l}\text { Polyenes-Amphotericin B, Nystatin, } \\
\text { Primaricin }\end{array}$ & $\begin{array}{l}\text { Membrane function-Binding } \\
\text { to ergosterol }\end{array}$ & $\begin{array}{l}\text { Absence of ergosterol or } \\
\text { decrease of ergosterol in } \\
\text { cells. }\end{array}$ \\
\hline $\begin{array}{l}\text { Azoles- Fluconazole, Itraconazole, } \\
\text { Voriconazole, Ketoconazole, } \\
\text { Posaconazole, Ravuconazole. }\end{array}$ & $\begin{array}{l}\text { Ergosterol synthesis- } \\
\text { Inhibition of } 14 \alpha \text {-lanosterol } \\
\text { demethylase (ERG11) }\end{array}$ & $\begin{array}{l}\text { Enhanced efflux mediated by } \\
\text { multidrug transporters } \\
\text { Decrease affinity in Erg } 11 p \\
\text { by mutation. } \\
\text { Upregulation of ERG11 } \\
\text { Alteration in the ergosterol } \\
\text { biosynthetic pathway. }\end{array}$ \\
\hline 5- Fluorocytosine (5-FC) & $\begin{array}{l}\text { DNA Synthesis-Inhibition of } \\
\text { DNA synthesis }\end{array}$ & Defect in cytosine permease \\
\hline $\begin{array}{l}\text { Cyclic lipopeptides- Caspofungin, } \\
\text { Micafungin }\end{array}$ & $\begin{array}{l}\text { Cell wall biosynthesis- } \\
\text { Inhibition of } \beta-1,3 \text { glucan } \\
\text { synthase }\end{array}$ & $\begin{array}{l}\text { Loss of } \beta-1,3 \text { glucan synthase } \\
\text { function. }\end{array}$ \\
\hline Allylamines- Terbinafine & $\begin{array}{l}\text { Ergosterol synthesis } \\
\text { Inhibition of squalene } \\
\text { epoxidase }\end{array}$ & Not yet reported. \\
\hline Morpholines-Amorolfine & $\begin{array}{l}\text { Ergosterol synthesis } \\
\text { Inhibition of sterol } \Delta^{14} \\
\text { reductase }\end{array}$ & Not yet reported. \\
\hline Griseofulvin & $\begin{array}{l}\text { Nuclear division-Binding to } \\
\text { tubulin }\end{array}$ & Not yet reported. \\
\hline
\end{tabular}

arginine with lysine at aminoacid 467 (abbreviated as R467K) has been found to be associated with azole resistance. Another point mutation was the replacement of threonine with alanine at position 315(T315A). This particular mutation was located in the active site pocket and led the reduction in azole binding. At least 12 point mutations have been reported to occur in azole-resistant clinical isolates. The mutations most frequently associated with azole resistance were $\mathrm{Y} 132 \mathrm{H}$, D278E, S405F, G464S and R467K. These substitutions/mutations leading to structural changes are sufficient to induce a decreased affinity to azoles (Vanduputte et al., 2005).

A common mechanism of resistance in azole resistance is gene amplification. The increase in the number of gene copies usually results in an increase in expression. Overexpression of ERG11 genes has been described in several clinical isolates. Increased levels of lanosterol demethylase were associated with resistant phenotype. The increased levels of protein were associated with ERG11 gene amplification and correlation with an increase in ERG11 mRNA levels. The gene amplification of ERG11 has been linked to a chromosome duplication, which results in overexpression of ERG11 as well as altered expression of a variety of other proteins. Resistance to fluconazole in many clinical isolates has often been associated with the transcriptional activation of ERG11. However it has been difficult to correlate the upregulation of the gene with the observed fluconazole resistance mainly due to the simultaneous occurrence of mutations or overexpression in ERG11 genes (White, 1998).

\section{Increased cellular drug efflux}

The most common mechanism of azole resistance has been mediated by enhanced drug-efflux mechanism. Candidal cells contain two types of efflux pumps known to contribute to drug resistance are ATP-binding-cassette $(A B C)$ transporters and major facilitator super-family transporters. Three genes with a defined role in efflux have been described in candida, two of them, CDR1 and $C D R 2$ codes for ABC transporters. The third gene MDR1 belongs to the major facilitator super family of transporters (MF). CDR1 and CDR2 seem to reduce accumulation of azoles whereas $M D R 1$ specifically reduces accumulation of fluconazole (White, 1998).

\section{ABC transporters of Candida}

ABCT are frequently associated with active efflux of cytotoxic molecules and are relatively hydrophobic or lipophilic as is the case with most azole drugs. ABCT of candida possess specific domains for membrane association, ATP binding and hydrolysis. It is composed of two homologous halves, each made of a hydrophilic, cytoplasmic nucleotide binding domain (NBD) and a transmembrane domain (TMD) represented by six 
Table 2: Factors, intermediates and inhibitors of fungal ergosterol biosynthetic pathway.

\begin{tabular}{|c|c|c|c|}
\hline \\
\hline \multicolumn{4}{|c|}{$\begin{array}{l}\text { ERGOSTEROL PATHWAY } \\
\text { Gene Name }\end{array}$} \\
\hline ERG 1 & Squalene epoxidase & Squalene & Allylamines \\
\hline & & 2, 3-Oxidosqualene & \\
\hline ERG 7 & Lanosterol synthase & $\underset{\text { Lanosterol }}{\downarrow}$ & \\
\hline ERG 11(CYP51) & Lanosterol 14-ademethylase & $\stackrel{\downarrow}{\downarrow}$ & Azoles \\
\hline ERG 24 & C-14 Sterol reductase & 4. 4-dimethvl zymosterol & Morpholines \\
\hline ERG 25 & C-4 Sterol methyloxidase & & \\
\hline ERG 26 & C-4 Sterol decarboxylase & & \\
\hline & & 4, 4-methyl zymosterol & \\
\hline ERG 27 & C-3 Sterol Ketoreducatse & & \\
\hline ERG 6 & C-24 Sterol methyl-transferase & Zymosterol & \\
\hline ERG 2 & C-8 Sterol isomerase & & Morpholines \\
\hline & & Episterol & \\
\hline ERG 3 & C-5 Sterol denaturase & Ergosta-5, 7, 24 (28) trienol & Azoles \\
\hline ERG 5(CYP61) & C-22 Sterol denaturase & $\stackrel{\downarrow}{\downarrow}$ & \\
\hline ERG 4 & C-24 Sterol reductase & $\underset{\text { Ergosterol }}{\downarrow}$ & \\
\hline
\end{tabular}

transmembrane segments (TMS). The characteristic of ABCTs is that they use ATP to transport the drugs across the membrane against the concentration gradient. The NBDs located at the cytoplasmic periphery possess specific Walker motifs $A$ and $B$, which aid in drug transport from the cytoplasm to cell membrane (White, 1998). Among all the putative ABCT proteins only $C D R 1$ and $C D R 2$ are experimentally implicated in azole resistance (White, 1997; Prasad, 2005;). Mechanism of functions of ABCTs of candida is shown in (Figure 2).

\section{Major facilitators (MFS)}

The MFS transporters are closely associated with hydrophilic molecules. Due to the diversity in nucleotide and amino acid sequences, the structure and functional relationships have not been generalized in detail. MFS protein utilizes a proton gradient to expel the drug. MFSs are composed primarily of 12 to 14 transmembrane segments, and uses membrane proton motive force as a source of energy, whereby protons are pumped into the cell and substrate molecules are pumped out (Figure 3 ).

Azoles enter the cell by an unknown mechanism and target lanosterol demethylase a product of the ERG11 gene, which is part of the ergosterol biosynthetic pathway. Low-level expressions of the $C D R$ and $M D R$ genes are frequently observed. In a resistant cell, the azoles are blocked from interacting normally with the target enzyme because the enzyme is modified and/or over expressed. Mutations in other enzymes in the pathway (such as ERG3) can contribute to resistance. Both ABCT (CDR gene) and MF (MDR gene) can be over expressed. The $C D R$ is responsible for resistance to many azole drugs while $M D R$ appears to be specific for fluconazole. 


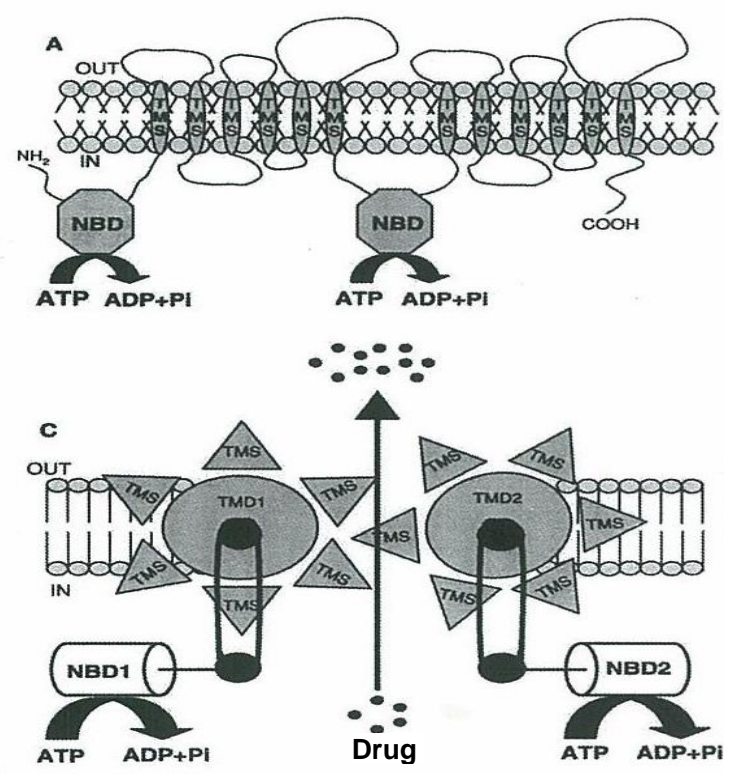

Figure 2: Diagrammatic representation of an $A B C$ transporter. NBD, Nucleotide-binding domain; TMS, Transmembrane segments; TMD, Transmembrane domain.

Azole resistance in $C$. albicans and $C$. glabrata was extensively reported but only few data are available for $C$. tropicalis. The azole resistance in $C$. tropicalis is a result of an increased efflux of azole drugs linked to the over expression of the CtMDR1 gene which encodes MFS protein, whereas CtCDR1 encoding ABC protein was not over expressed and that the increased efflux is the most frequent mechanism involved in azole resistance, with an overexpression of CtERG11 associated with a missense mutation of this gene. A similar study was described by Vanduputte et al. and Chong et al. and showed an increased gene expression in CDR1, MDR1 gene and also detected point mutations in CDR1, MDR1 but not in ERG11 gene (Chong et al., 2006).

\section{Enterobacterial repetitive intergenic consensus (ERIC)}

Molecular or genotypic methods have received considerable attention and as a means for analyzing epidemiological interrelationship in order to study the epidemiology of candida infections (Sewell et al., 1994; Deak et al., 2000). Repeat primer PCR achieves taxonomy, confirmation of strain identity and direct epidemiological identification of strains. DNA primers complementary to naturally occurring repetitive DNA sequences dispersed among many microorganisms are used in rep-PCR genomic finger printing like REP (Repetitive Extragenic Palindrome) sequence, ERIC (Enterobacterial Repetitive Intergenic Consensus) sequence (Sewell et al., 1994).
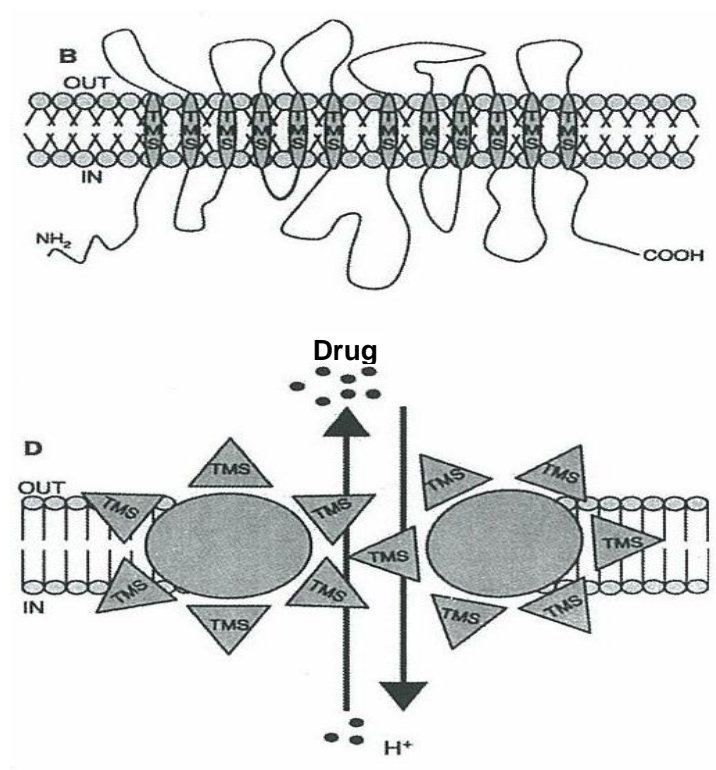

Figure 3: Diagrammatic representation of MFS transporters. TMD, Transmembrane domain.

ERIC sequences described as intergenic repetitive units was originally developed for typing $M$. tuberculosis and has been useful for differentiating strains of enterobactericeae members. These sequences have 127 bp imperfect palindromes that occur in multiple copies in the genomes of enteric bacteria. They have been used as the basis technique for fingerprinting. PCR-primers were designed to amplify between copies of the ERIC sequence at nearby locations. This ERIC-PCR approach has subsequently been very widely used to analyze a very broad range of species (Deak et al., 2000). ERIC sequences are highly conserved at the nucleotide sequence level, but their chromosomal locations differ among species and strains. The elements have been successfully used for molecular typing purposes. By use of ERIC-PCR differences in band sizes representing polymorphism due to repetitive elements in different genomes can be analyzed for developing phylogenetic relationships. This also allows the identification of interstrain genotypic diversity (Chieng et al., 2006).

\section{CONCLUSION}

The incidence of life threatening fungal infections due to Candida spp. has increased markedly over the past two decades. C. tropicalis is an important pathogenic Candida spp. and has been reported to be a common cause of fungemia in both oncology and non-oncology patients. It is most commonly recovered from blood culture in cancer patients. In neutropenic patients, $C$. tropicalis has the potential to cause severe invasive disease and hematogenous infection which has been associated with a 
high mortality rate. Antifungal treatment of Candida infections are hampered by several factors, including the limited number of active agents, the emergence of refractory fungal species, and the development of resistance. This should lead to the search for new antifungal agents with novel modes of action.

\section{REFERENCES}

Arikan, S. and Rex, J. H. (2005). Resistance to antifungal agents. Medical Mycology Topley and Wilson $10^{\text {th }}$ Edn. ASM Press. pp. 168-177.

Azoulay, E., Timsit, J. F. and Tafflet, M. (2006). Candidal colonization of the respiratory tract and subsequent Pseudomonas ventilator-associated pneumonia. Chest 129, 110-117.

Bernardis, D. F., Chiani, P., Ciccozzi, M., Pellegrini, G., Ceddia, T., D’Offizzi, G., Quinti, I., Sullivan, P. A. and Cassone, A. (1996). Elevated aspartyl proteinase secretion and experimental pathogenicity of Candida albicans isolates from oral cavities of subjects infected with human immunodeficiency virus. Infection and Immunity 64, 466-471.

Bernard, H. and Naglik, J. (2002). Candida and Candidiasis. ASM Press. pp. 107-122.

Bradly, N. and Richard, D. (1991). Restriction fragment analysis of a Candida tropicalis outbreak of sternal wound infections. Journal of Clinical Microbiology 29, 1268-1270.

Cannon, R. D., Lamping, E., Holmes, A. R., Niimi, K., Baret, P. V., Keniya, M. V., Tanabe, K., Niimi, M., Goffeau, A., and Monk, B. C. (2009). Efflux-mediated antifungal drug resistance. Clinical Microbiology Review, 22, 291-321.

Carlos, V. and Paya. (1993). Fungal infections in solid organ transplantation. Clinical Infectious Diseases 16, 677-688.

Cassone, A., De Bernardis, F., Torosantucci, A., Tacconelli, E. and Tumbarello, M. C. R. (1999). In vitro and in vivo anticandidal activity of human immunodeficiency virus protease inhibitors. Journal of Infectious Diseases 180, 448-453.

Cheng, G., Yeater, K. M. and Hoyer, L. L. (2006). Cellular and molecular biology of Candida albicans estrogen response. Eukaryot Cell 5, 180-191.

Chieng, D., Chong, P. P., Low, L. Y., Hafeez, A. and Mariana, N. S. (2006). Recurrent candidemia in a neonate with Hirschsprung's disease: Fluconazole resistance and genetic relatedness of eight Candida tropicalis isolates. Journal of Medical Microbiology 55, 423-428.

Chong, P. P., Yong, V. C., Chew, L. M., Kwan, C. L., Ng, K. P. and Seow, H. F. (2006). Transcriptional and sequencing analysis of CtCDR1, CtMDR1 and CtERG11 genes in fluconazole-resistant Candida tropicalis isolates from candidemia patients. Journal of Medical Science 6, 713-723.

Christelle, F. and Bradley, N. (1994). Candida tropicalis vertebral osteomyelitis: A late sequel of fungemia. Clinical Infectious Diseases 19, 697-703.
Cohen, M. and Montgomerie, Z. (1993). Hematogenous endophthalmitis due to Candida tropicalis: Report of two cases and review. Clinical Infectious Diseases 17, 270-272.

Deak, T., Chen, J. and Beuchat, L. R. (2000). Molecular characterization of Yarrowia lipolytica and Candida zeylanoides isolated from poultry. Applied Environmental Microbiology 66, 4340-4344.

Dorko, E., Kmetova, M. and Skardova, I. (2000). Incidence of Candida tropicalis in clinical samples over 5 -year period. Pakistan Journal of Biological Sciences 3, 606-609.

Eschenauer, G., DePestel, D. D. and Carver, P. L. (2007). Comparison of echinocandin antifungals. Therapeutic and Clinical Risk Management 3, 71-97.

Garcia-Effron, G., Lee, S., Park, S., Cleary, J. D. and Perlin, D. S. (2009). Effect of Candida glabrata FKS1 and FKS2 mutations on echinocandin sensitivity and kinetics of 1,3- $\beta$-D-glucan synthase: Implication for the existing susceptibility breakpoint. Antimicrobial Agents Chemotherapy 53, 3690-3699.

Gary, P. M., Sullivan, D. J. and Coleman, D. (2002). Candida and Candidiasis. ASM Press. pp. 37-53.

Gerritsen, J. G., Dissel, J. T. and Verwey, H. F. (1998). Candida tropicalis endocarditis. Circulation 98, 90-91.

Ghannoum, M. (2000). Potential role of Phospholipases in Virulence and fungal Pathogenesis. Clinical Microbiology Review 13, 122-143.

Girish Kumar, C. P., and Thangam, M. (2006). Biofilm production by clinical isolates of Candida species. Medical Mycology 44, 99-101.

Groll, A. H. and Walsh, T. J. (2001). Uncommon opportunistic fungi: New nosocomial threats. Clinical Microbiology and Infection 7, 8-24.

Hashimoto, S. (2009). Micafungin: A sulfated echinocandin. Journal of Antibiotics 62, 27-35.

Hawser, S. P. and Douglas, J. (1995). Resistance of Candida albicans biofilms to antifungal. Antimicrobial Agents and Chemotherapy 39, 2128-2131.

Jabra, M. A. R., Falkler, W. A. and Meiller, T. F. (2004). Fungal biofilms and drug resistance. Emerging Infectious Diseases 10, 14-19.

Julia, D. L. (2002). Medical importance of biofilms in Candida infections. Revistan Iberoamericana de Micologica 19, 139-143.

Jyotsna, C., Duncan, M. K., Mukherjee, P. K., Hoyer, L. L., McCormick, L. and Ghannoum, M. A. (2001). Biofilm formation by the fungal pathogen Candida albicans: Development, architecture and drug resistance. Journal of Bacteriology 183, 5385- 5394.

Kontoyiannis, D., Vaziri, I. and Hanna, H. A. (2001). Risk factors for Candida tropicalis fungemia in patients with cancer. Clinical Infectious Diseases 33, 16761681.

Kullberg, B. and Filler, S. G. (2002). Candida and Candidiasis. ASM Press. pp. 327-340.

Loeffler, J. and Stevens, D. A. (2003). Antifungal drug resistance. Clinical Infectious Diseases 36, S31-S41. 
Lupetti, A., Danesi, R., Campa, M., Tacca, M. D. and Kelly, S. (2002). Molecular basis of resistance to azole antifungals. Trends in Molecular Medicine 8, 76-81.

Martín, V., García, B., Carnero, E., Durán, A. and Sánchez, Y. (2003). Bgs3p, a putative 1,3- $\beta$-glucan synthase subunit, is required for cell wall assembly in Schizosaccharomyces pombe. Eukaryot Cell 2, 159169.

Mary, S., Pramila, R. and Suresh, M. (2001). Emergence of Candida tropicalis as the major cause of fungemia in India. Mycoses 44, 278-280.

Saccente, M. (2003). Clinical mycology. Churchill Livingstone. pp. 383-396.

Michael, B. and Edmond. H (1999). Nosocomial Blood stream infections in United States Hospitals: A Three year analysis. Clinical Infectious Diseases 29, 239244.

Munn, A. L., Heese-Peck, A., Stevenson, B. J., Pichler, H. and Riezman, H. (1999). Specific sterols required for the internalization step of endocytosis in yeast. Molecular Biology and Cell 10, 3943-3957.

Nafsika, H., Georgopapadakou B, and Walsh, T. J. (1996). Antifungal agents: Chemotherapeutic targets and immunologic strategies. Antimicrobial Agents and Chemotherapy 40, 279-291.

Nguyen, H. and Yu, V. L. (1995). Meningitis caused by Candida species: An emerging problem in neurosurgical patients Clinical Infectious Diseases 21, 323-327.

Peter, G., Pfaller, M. A., Pappas C, and Wingard, R. (2006). Invasive fungal pathogens: Current epidemiological trends. Clinical Infectious Diseases 43, S3-S14.

Pfaller, M. A. and Diekema, D. J. (2007) Epidemiology of invasive candidiasis: A persistent public health problem. Clinical Microbiology Review 20, 133-163.

Prasad, R. and Khyati, K. (2005). Multidrug resistance in yeast Candida. International Review of Cytology 242, 215-248.

Price, M. F., Wilkinson, D. I., Layne, O. and Gentry. (1982). Plate method for defection of phospholipase activity in Candida albicans. Sabouraudia 20, 7-14.

Ramage, G., Bachmann, S., Patterson, T., Brinan, F., Wickes, L. and Jose, L. (2002). Investigation of multidrug efflux pumps in relation to fluconazole resistance in Candida albicans biofilms. Journal of Antimicrobial Chemotherapy 49, 973-980.

Ramage, G., Walle, K. C., Wickes, B. L. and Jose, L. (2001). Biofilm formation by Candida dubliniensis. Jounal of Clinical Microbiology 39, 3234-3240.

Roilides, E., Farmaki, E. and Evdoridou, J. (2003). Candida tropicalis in a neonatal intensive care unit: Epidemiologic and molecular analysis of an outbreak of infection with an uncommon neonatal pathogen. Journal of Clinical Microbiology 41, 735-741.

Rudek, W. (1978). Esterase activity in Candida species. Journal of Clinical Microbiology 8, 756-769.

Runkhe, M. (2002). Candida and Candidiasis ASM Press. pp. 307-326.
Sanglard, D. and Bille, J. (2002). Candida and Candidiasis. ASM Press. pp. 349-383.

Seneviratne, C. J., Jin, L. J., Samaranayake, Y. H., and Samaranayake, L. P. (2008). Cell density and cell aging as factors modulating antifungal resistance of Candida albicans biofilms. Antimicrobial Agents Chemotherapy 52, 3259-3266.

Sewell. D. L., Pfaller, M. A. and Barry, A. L. (1994). Comparison of Broth macro dilution, Broth microdilution and Etest antifungal susceptibility tests for fluonazole. Journal of Clinical Microbiolgy 32, 20992102.

Shankar, E. M., Kumarasamy, N., Bella, D., Renuka, S., Kownhar, H., Suniti, S., Rajan, R. and Rao, U.A. (2006). Pneumonia and pleural effusion due to Cryptococcus laurentii in a clinically proven case of AIDS. Canadian Respiratory Journel 13, 275-278.

Shankar, E. M., Vignesh, R., Barton, R. C., Balakrishnan, P., Murugavel, K. G., Velu, V., Kownhar, H., Lloyd, C. A., Solomon, S., Rao, U. A., and Kumarasamy, N. (2007). Hydrothorax in association with Scopulariopsis brumptii in an AIDS patient in Chennai, India. Transactions of Royal Society of Tropical Medicine and Hygiene 101, 12701272.

Sim, J. P. Y., Kho, B. C. and Chan, J. C. W. (2005). Candida tropicalis arthritis of the knee in a patient with acute lymphoblastic leukemia successful treatment with caspofungin. Hong Kong Medical Journal 11, 120123.

Slifkin, M. (2000). Tween 80 Opacity test responses of various Candida species. Journal of Clinical Microbiology 38, 4626-4628.

Stehr, F., Kretschmar, M., Kroger, C., Hube, B. and Schafer, W. (2003). Microbial lipases as virulence factors. Journal of Molecular Catalysia and $B$ : Enzymatic 22, 347-353.

Vanduputte, P., Larcher, G. and Berges, T. (2005). Mechanism of azole resistance in clinical isolate of Candida tropicalis. Antimicrobial Agents and Chemotherapy 49, 4608-4615.

Walsh, T. J. (1995). Fungemia in children infected with the human immunodeficiency virus: New epidemiologic patterns. Emerging Pathogens, and improved outcome with Anti fungal Therapy. Clinical Infectious Diseases 20, 900-906.

Wenzel, R. P. and Pfaller, M. A. (2003). Clinical Mycology. Churchill Livingstone. pp. 3-19.

White, T. C., Kieren, A., Raleigh, A. and Bowden. (1998). Clinical, cellular and molecular factors that contribute to antifungal drug resistance. Clinical Microbiology Review 11, 382-402.

White, T. C. (1997). Increased mRNA levels of ERG16, $C D R$, and MDR1 correlates with increases in azole resistance in Candida albicans isolates from an HIV infected patient. Antimicrobial Agents and Chemotherapy 41, 1482-1487.

Wingard, J. R. (1995). Importance of Candida species other than $C$. albicans as pathogens in oncology patients. Clinical Infectious Diseases 20, 115-125. 